\title{
Top Quark Production at Tevatron
}

\section{Yen-Chu Chen ${ }^{1}$}

Fermil National Accelerator Laboratory

Kirk and Wilson, Batavia, IL 60510, USA

E-mail: chenyc@fnal.gov

\section{For the CDF and DO collaborations}

The top quark was discovered at the Tevatron by the CDF and D0 experiments in 1995. Until the conclusion of the Tevatron in Sep. 2011, each experiment collected approximately $10 \mathrm{fb}^{-1}$ of proton-antiproton collisions. We report the latest measurements of the $t \bar{t}$ production cross section, and update the studies of the recently discovered forward-backward asymmetry in the angular distributions of $t$ and $\bar{t}$ quarks in events corresponding to the full integrated luminosity.

The XIth International Conference on Heavy Quarks and Leptons,

Prague, Czech Republic

June 11-15, 2012

$1 \quad$ Speaker 


\section{Introduction}

The top quark was discovered in 1995 by CDF and D0 experiments at Tevatron [1][2]. Since then much effort has gone into studying this heaviest particle in the Standard Model (SM). To date, most of the results agree well with SM predictions, with possible exception of the production asymmetry, which states that the emmitted top quark is more along the direction of proton beam rather than the antiproton beam.

The top quark decays into a $W^{+}$boson and $b$ quark, while antitop quark decays into $W$ and anti- $b$ quark. Depending on how the $W$ bosons decay the events are selected into the lepton plus jets (LJ) channel, where only one $W$ boson decays leptonically, and di-lepton (DIL) channel, where both $W$ bosons decay leptonically. In the following sections results of top pair production cross section and the production asymmetry are reported from both CDF and D0 experiments for both LJ and DIL channels.

\section{Event selection}

In both LJ and DIL channels there are, respectively, either one or two neutrinos missing in the detection, which causes apparent imbalance in momentum thus high missing transverse energy. Requiring leptons ( $e$ or $\mu$ ) with high transverse momentum $\left(P_{T}\right)$ and jets with high transverse energy $\left(E_{T}\right)$ as well as high missing transverse energy provides effective rejection of the multi jets (MJ) background.

At CDF (D0) leptons are required to have $P_{T}$ larger than 20 (15) GeV/c. Both experiments require that the primary leptons are in the central region, $|\eta|<2$ or smaller, where $\eta$ is pseudo rapidity. The $E_{T}$ of selected jets must be larger than $20 \mathrm{GeV}$ in both experiments.

Because the top pair system has large mass there are high energy leptons, jets and neutrinos. There should be high total transverse energy, $H_{T}$, which is the scalar sum of $P_{T} / E_{T}$ of the leading leptons, jets and the missing transverse energy. At CDF it is required to be greater than $200 \mathrm{GeV}$ in the DIL channel [3]. At D0 the $\mathrm{H}_{\mathrm{T}}$ doesn't include the missing transverse energy. For the $e \mu$ events, $H_{T}$ should be larger than $105 \mathrm{GeV}$ or $110 \mathrm{GeV}$ for one or two jets in a given event respectively. For the ee events, it is required that significance of the imbalanced momentum should be larger than 5. Similar requirement is made in selecting $\mu \mu$ events; in addition there is requirement of imbalanced momentum being greater than $40 \mathrm{GeV} / c$ [4] .

To minize the background from MJ events, it is useful to require that at least one jet is from $b$ quark decay. Since $\mathrm{b}$ quarks have long life time, the decay points of their hadrons can be reconstructed as the secondary vertices from the tracks within the $b$ jet downstream of the primary vertex. This is refered to as SecVtx $b$ tagging at CDF. For the LJ channel, this is essential but for DIL events this is useful only to provide ultra pure samples of the top quark pairs.

In case of DIL events, one dominant background is from $Z$ boson decaying to $e e$ or $\mu \mu$. To select the DIL top pair candidates it is therefore important to suppress $Z$ boson events. In a DIL top event both $W$ bosons decay leptonically so there is large missing transverse energy due to the two neutrinos. On the other hand, for $Z$ boson decay, the missing transverse energy comes 
from mismeasurement of jet energy. At CDF, the suppressing of $Z$ boson background is accomplished by requiring a significance of greater than 4 standard deviations on missing transverse energy, when the invariant mass of $e e$ or $\mu \mu$ are within the mass range of $Z$ boson, 76 $\mathrm{GeV} / \mathrm{c}^{2}<m_{l l}<106 \mathrm{GeV} / \mathrm{c}^{2}$. At D0 the suppresion is realized through a neural network (NN) that is also based on the significance of the missing transverse energy.

The DIL events have to have two or more jets due to the two $b$ quarks in the $t \bar{t}$ final state. For the LJ events there are four or more jets due to the two $b$ quarks and the two quark jets from the decay of one of the $W$ bosons.

\section{Top production cross section}

The latest theoritical prediction shows that top quark pair production is $7.22^{+0.31}{ }_{-0.34}$ (total theoritical ${ }^{+0.71}{ }_{-0.55}\left(\mathrm{PDF}+\alpha_{\mathrm{s}}\right) \mathrm{pb}$ at $\sqrt{\mathrm{S}}=1.96 \mathrm{TeV}$ based on Next to Next Leading Logrithmic (NNLL) [5]. The fraction of quark anti-quark annihilation and gluon gluon fusion are estmated to be $90 \%$ and $10 \%$, respectively.

From CDF the latest result is based on the DIL samples, corresponding to a total luminosity of $5.1 \mathrm{fb}^{-1}$. In the near future this will be updated with the full set of data corresponding to about $10 \mathrm{fb}^{-1}$.

The distribution of the number of jets per event in the final sample of DIL top candidates is shown in the Figure 1, left side. There is good agreement between the data and a Monte-Carlo (MC) estimation based on top quark mass of $m_{t}=172.5 \mathrm{GeV} / \mathrm{c}^{2}$. The measured cross section is $\sigma_{\text {tt }}=7.40 \pm 0.58_{\text {stat }} \pm 0.63_{\text {syst }} \pm 0.45_{\text {lumi }} \mathrm{pb}$.
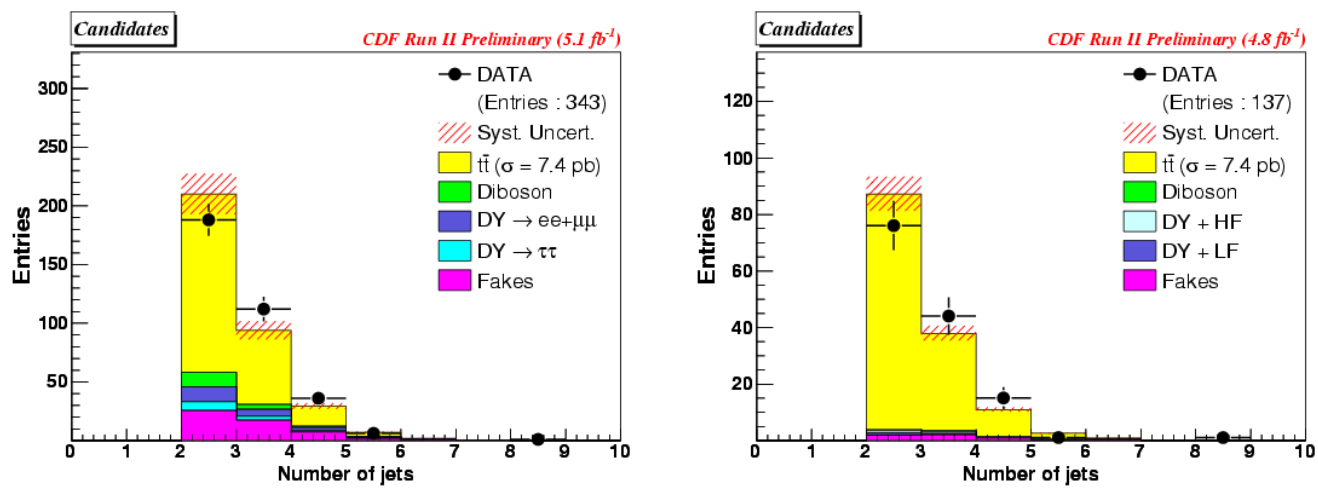

Figure 1: These two histograms show the distribution of number of jets in the DIL top candidates from CDF. The histogram in the left side has no requirement of $b$ tagging; the one in the right side is after the requiring at least on jet is $b$ tagged. The data show good agreement with estimates based on MC simulation of top signal and known background types as well as estimation of fake leptons from data.

Requiring at least one jet being $b$ tagged can reject the majority of the background for a loss of $40 \%$ in signal efficiency. Figure 1 right side histogram shows the distribution of number of jets for the DIL events selected with additional requirement of having at least one $b$ tagged jet. The agreement between data and estimation is very good and the measured cross section from 
these $b$-tagged events is $\sigma_{t t}=7.25 \pm 0.66_{\text {stat }} \pm 0.47_{\text {syst }} \pm 0.44_{\text {lumi }} \mathrm{pb}$. The two measurements from the DIL samples before and after requiring $b$ tagging are in good agreement.

A summary of the latest measurements of the top production cross section is given in Figure 2. The LJ channel is being studied and is expected to be updated during summer of 2012.

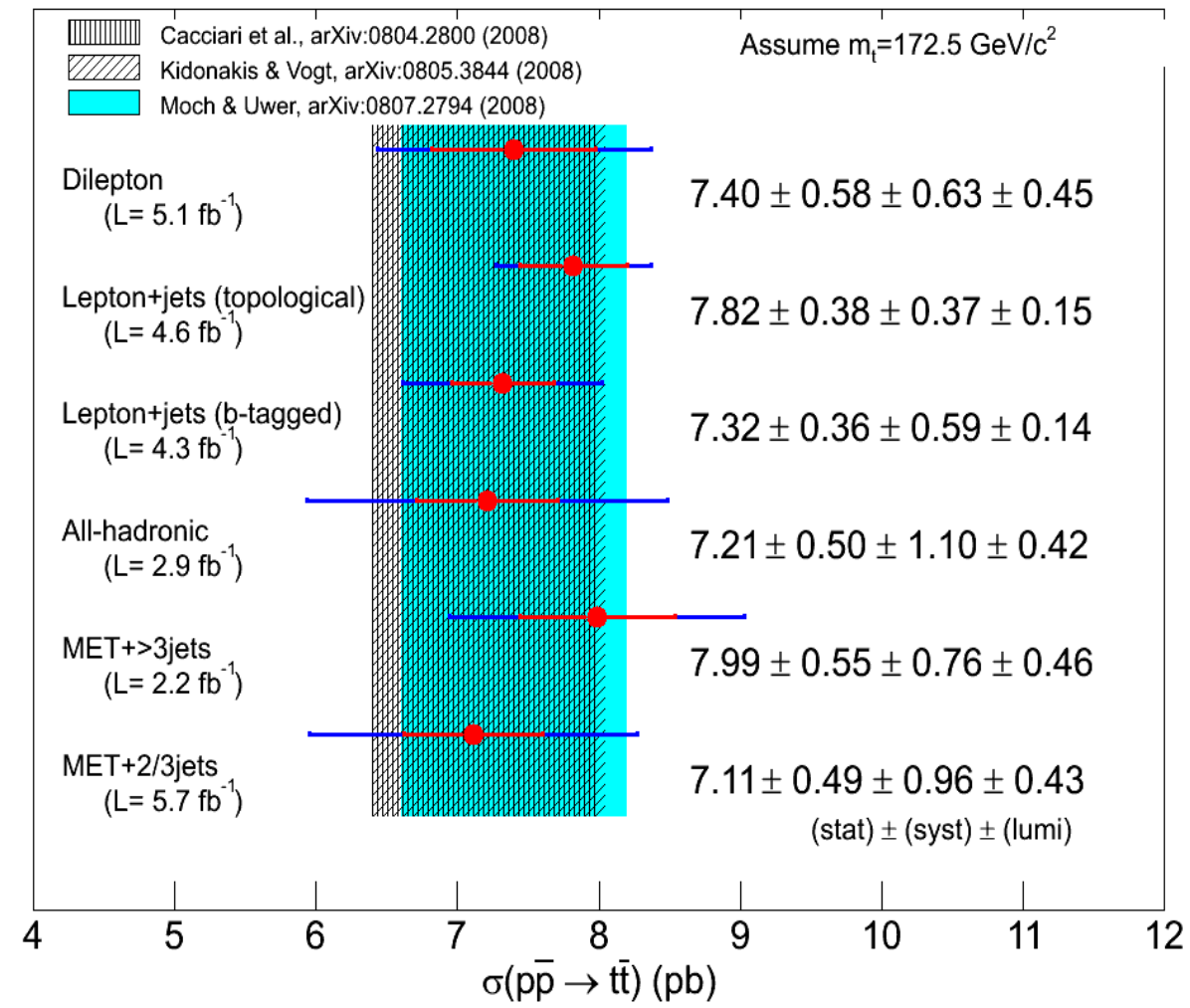

Figure 2: Comparison of theory with data for the top quark production cross sections measured in different channels at the CDF experiment shows agreement. An update using the full luminosity will be available in the near future.
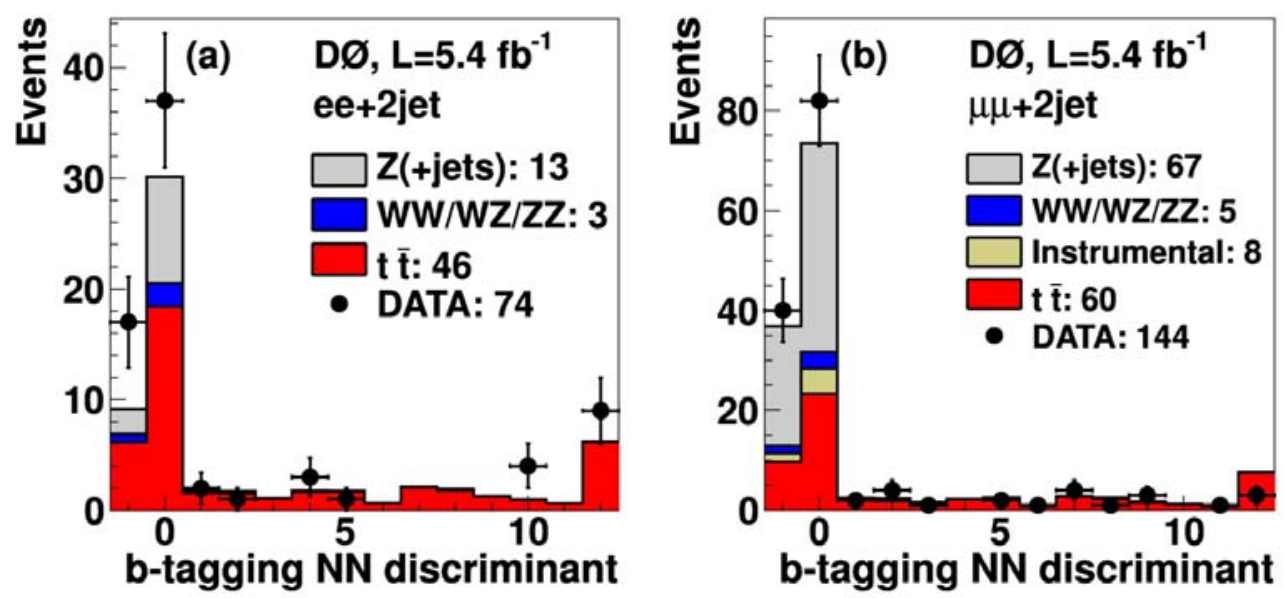

Figure 3: The distributions of the $\mathrm{NN}$ discriminants for $e e+2$ jets, $\mu \mu+2$ jets channels from the D0 experiment, with $b$ tagging required on at least one jet. 

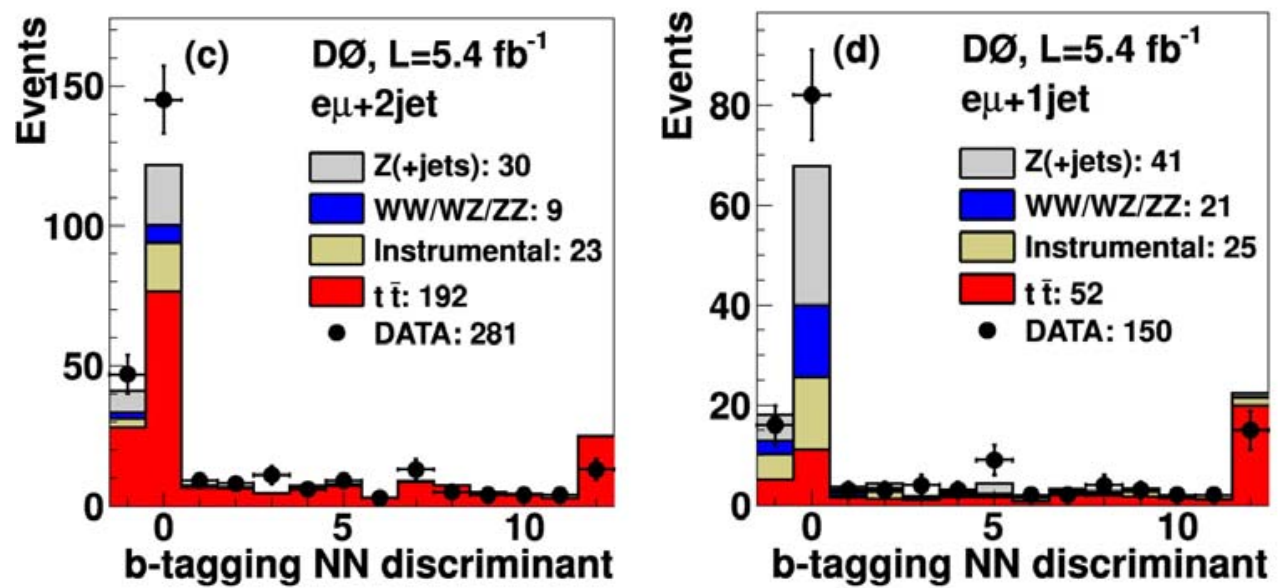

Figure 4: The distributions of the NN discriminants for $e \mu+2$ jets, $e \mu+1$ jet channels from the D0 experiment, with $b$ tagging required on at least one jet.

DØ Run II

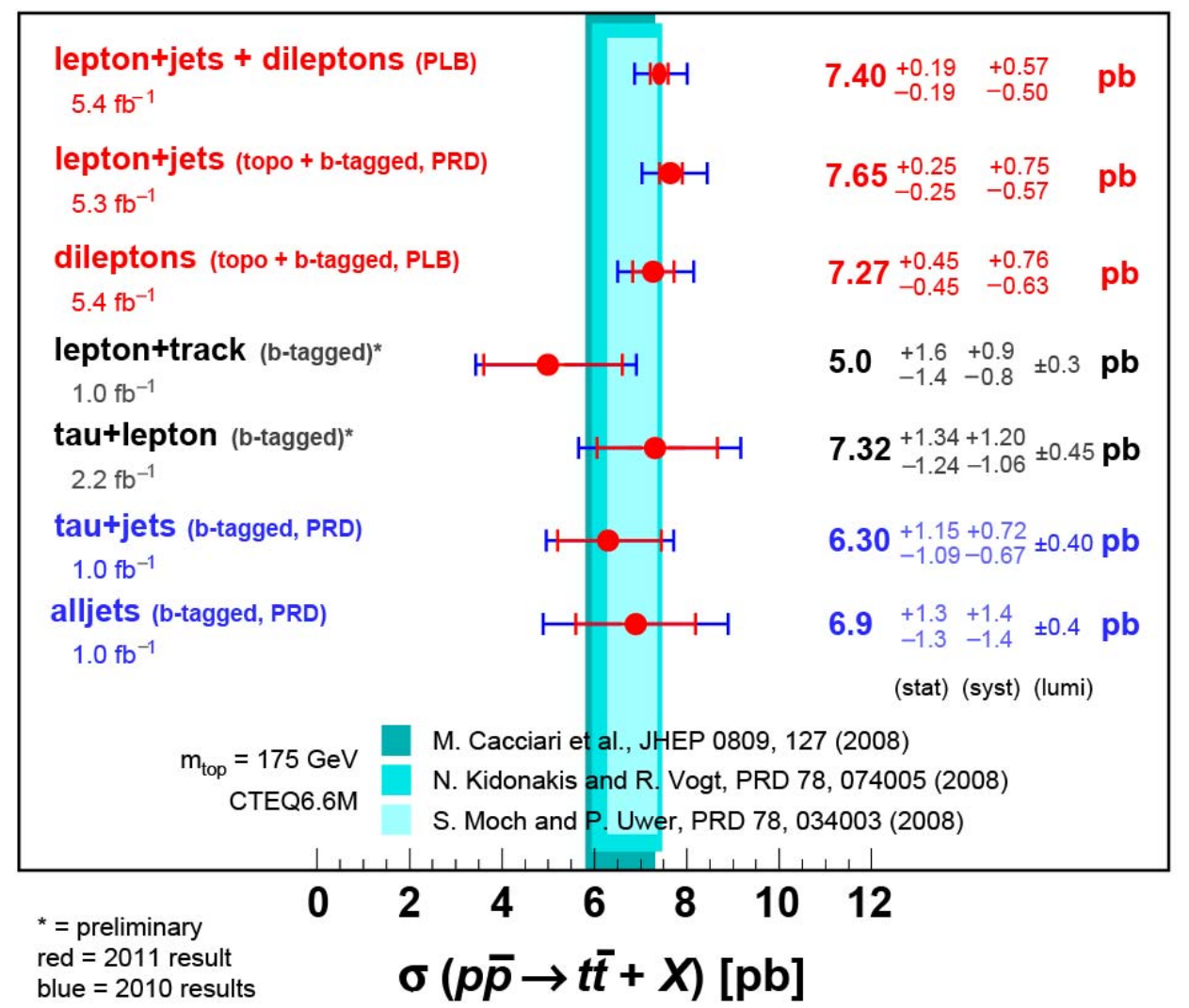

Figure 5: Comparison of theory with data for the top quark production cross sections measured in different channels at the D0 experiment shows agreement. An update using the full luminosity will be available in the near future. 
At D0, $b$ tagging on at least one jet is required for DIL analysis, and a neural network is used to separate the top candidates from background. Figures 3 and 4 show, respectively, the distributions of the NN discriminants for the $e e+2$ jets, $e \mu+2$ jets, $\mu \mu+2$ jets and $e \mu+1$ jet events. Where the expectation of top signal is based on top mass of $m_{t}=172.5 \mathrm{GeV} / \mathrm{c}^{2}$. The cross section is measured to be $\sigma_{t t}=7.36^{+0.90}{ }_{-0.79}$ (stat,syst,lummi and added in quardrature) pb. Combined with the result from LJ, the result is $\sigma_{t t}=7.56^{+0.63}{ }_{-0.56} \mathrm{pb}$ [6]. A summary from D0 experiment is shown in Figure 5.

\section{Production angular asymmetry}

The dependence of the top or antitop production angle in the $t \bar{t}$ rest frame relative to the beam provides an interesting test of QCD; and the differential cross section in $\cos \theta^{*}$ is expected to depand on variables such as the $P_{T}$ of the top pair system and its mass $M_{t t}$. In the experiment we use $\Delta y$, the rapidity difference of the top and antitop quark to examine the dependence on $\cos \theta^{*}$.

The variable $\Delta y$ reflects the forward/backward asymmetry in the angular distributions of top and antitop quarks, which must be identical because of CP symmetry of the $p \bar{p}$ system. Surprisingly the distribution of $\Delta y$ indicates the presence of an angluar asymmetry larger than expected from perturbative QCD from both CDF [7] and D0 [8]. Although the significance of the deviation from the prediction is less than 3 standard deviatioin, this effect has attracted much attention, e.g. in Ref. [9] and references therein. In this report we present the latest result using the full CDF set of data from the LJ channel.

For the full data, with the requirement that events are used only when the silicon detector was operating well, CDF reports a total luminosity of $8.7 \mathrm{fb}^{-1}$. After event selections there are 2498 top candidate events, among which $505 \pm 123$ are estimated to be from background, mostly from the $W+$ jets. Based on data of $5.4 \mathrm{fb}^{-1}$ luminosty D0 has 1532 events with 435 events from $W+$ jets and multijet production [9].

Accepted top candidate events are reconstructed in a kinematic fit of the data to a $\boldsymbol{t} \overline{\boldsymbol{t}}$ hyposthesis, assuming a top mass of $172.5 \mathrm{GeV} / \mathrm{c}^{2}$. All possible jet combinations are tried to form the "leptonic" top quark (the system having $W$ decays leptonically) and the "hadronic" top quark (the system having $W$ decay hadronically). The combination with best $\chi^{2}$ is chosen to represent the event. Shown in Figure 6 are the $\chi^{2}$ distributions from reconstruction of top events for both CDF (left) and D0 (right). The agreement between the data and the simulation is very good.

Figure 7 shows the invariant mass distribution of the reconstructed $t \bar{t}$ quark pairs from $\mathrm{CDF}$. The data agrees very well with the prediction from the SM.

For D0, shown in Figure 8 is the distribution of the invariant mass of the two jets that are not assigned to be $b$ quarks. The data agrees very well with the prediction from the SM. 

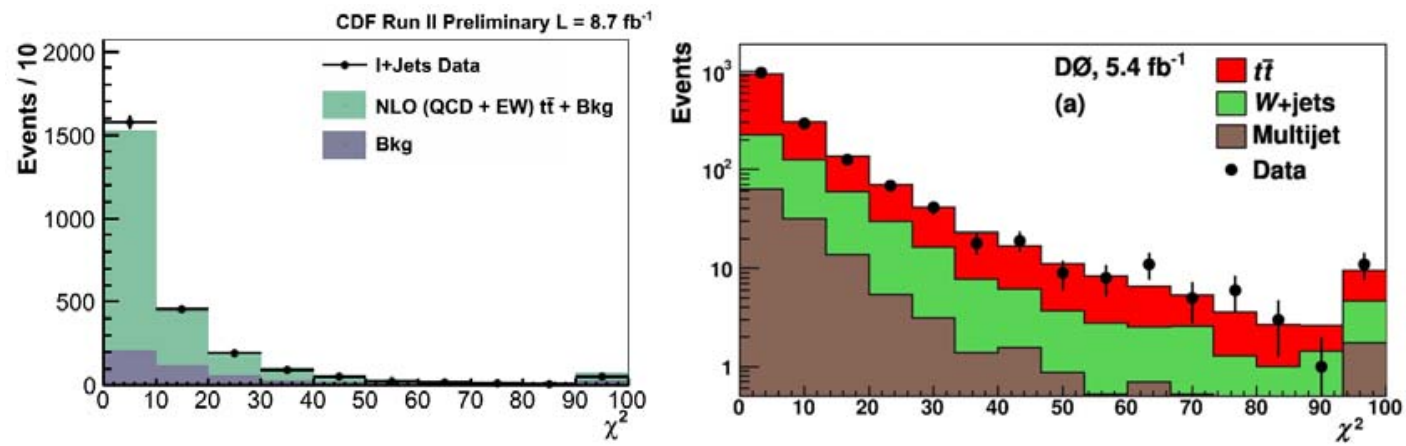

Figure 6: The $\chi^{2}$ distributions from the reconstruction of top events for both CDF (left) and D0 (right).

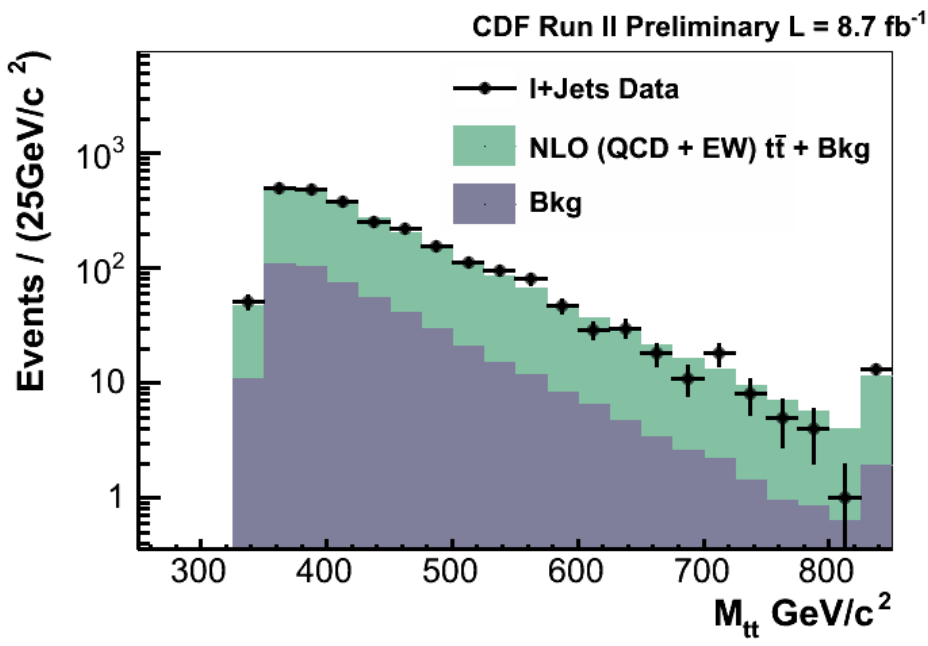

Figure 7: The distribution of the invariant mass of the reconstructed top quark pairs from CDF. The agreement between the data and the prediction from SM is very good.

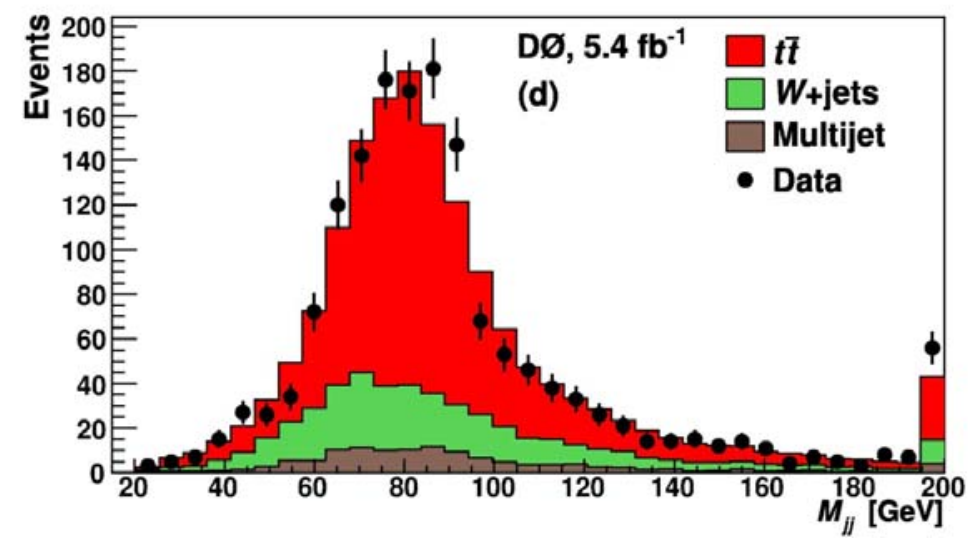

Figure 8: The distribution of the invariant mass of the two jets from the $W$ boson following the top event reconstruction in D0. The agreement between data and prediction from SM is very good. 
The event reconstrution provides the momentum of the top and antitop quark, from which their rapidity difference, $\Delta y$, can be calculated. We define the forward-backward asymmetry of the top and antitop system by:

$$
\begin{aligned}
A_{F B}^{t \bar{t}} & =\frac{N(\Delta y>0)-N(\Delta y<0)}{N(\Delta y>0)+N(\Delta y<0)} \\
& =\frac{N\left(y_{t}^{t \bar{t}}>0\right)-N\left(y_{t}^{t \bar{t}}<0\right)}{N\left(y_{t}^{t \bar{t}}>0\right)+N\left(y_{t}^{t \bar{t}}<0\right)}
\end{aligned}
$$

where $N(\Delta y>0)$ and $N(\Delta y<0)$ are the the numbers of events having $\Delta y>0$ and $\Delta y<0$ respectively; this is equivalent to the asymmetry in the top quark production angle in the $\boldsymbol{t} \overline{\boldsymbol{t}}$ rest frame, where $y_{t}^{t \bar{t}}$ is the rapidity of top quark in the $t \bar{t}$ rest frame.

The distribution of reconstructed $\Delta y$ from CDF and D0 are shown in Figure 9. Both CDF and D0 observe systematically more events with $\Delta y>0$ than for $\Delta y<0$. The $t \bar{t}$ simulation is based on an NLO calculation using contributions from QCD and EW processes. The final estimate includes expectations from background. There is a clear asymmetry, which deviates from the SM prediction by $\sim 2$ standard deviation after event reconstruction, top (antitop) quark emitted along the proton (antiproton) direction than expected from theory. The asymmetry from the background is very small.
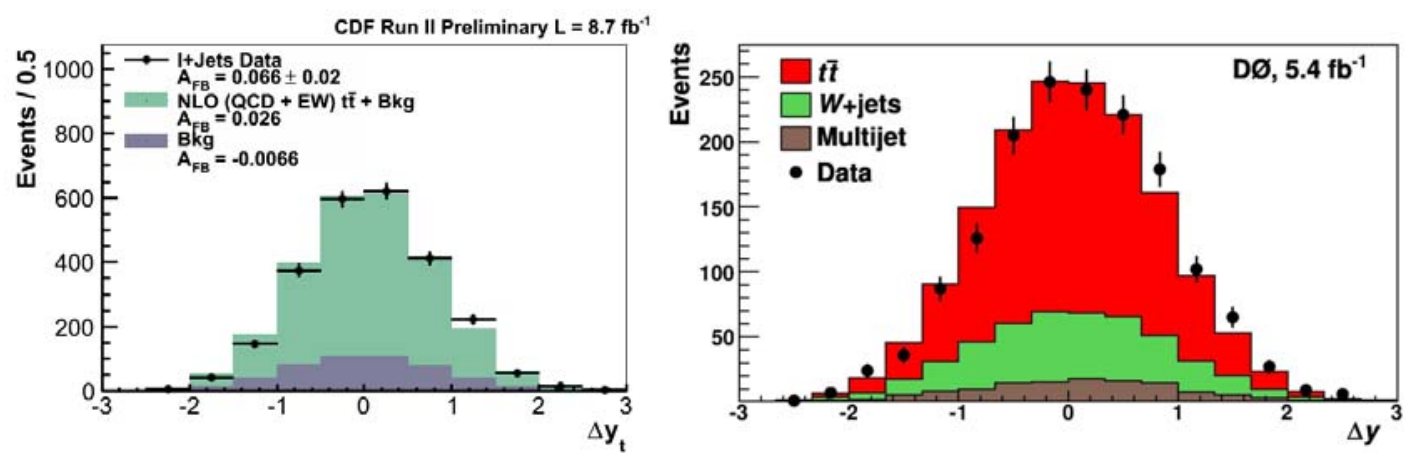

Figure 9: The distributions in $\Delta y$ for the reconstructed LJ $t \bar{t}$ events from CDF (left) and D0 (right). Both distributions show more events with $\Delta y>0$ than those with $\Delta y<0$.

Figure 10 shows the dependence of $A_{F B}$ on $\Delta y$ from CDF after the background subtraction. In each bin events having absolute $|\Delta y|$ value in the $\Delta y$ bin boundary are selected. Asymmetry is calculated for these events in this bin using $E q$ (1). In the region where $\Delta y$ is small there is consistency between data and the SM prediction. However in the region where $\Delta y>1$ the data are systematically above the prediction.

The asymmetry as a function of $M_{t t}$ is shown in Figure 11 for the CDF data, after the background subtraction. It is only in the lowest mass bin where there is consistency between data and prediction from the SM. In the region, where of $M_{t t}>450 \mathrm{GeV} / \mathrm{c}^{2}$, the data are 
systematically higher than the prediction. However this dependence on $M_{t t}$ is less significant at D0.

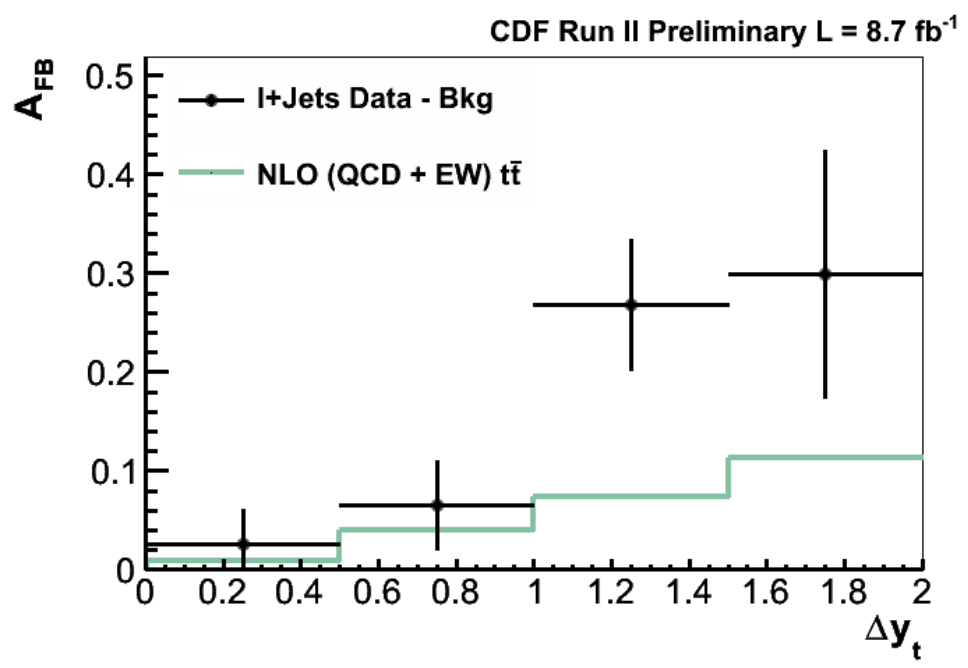

Figure 10: Dependence of $A_{F B}$ on $\Delta y$ after the background subtraction. The two data points with smaller $\Delta y$ are consistent with the SM prediction. However at higher $\Delta y$ the data are higher than expected.

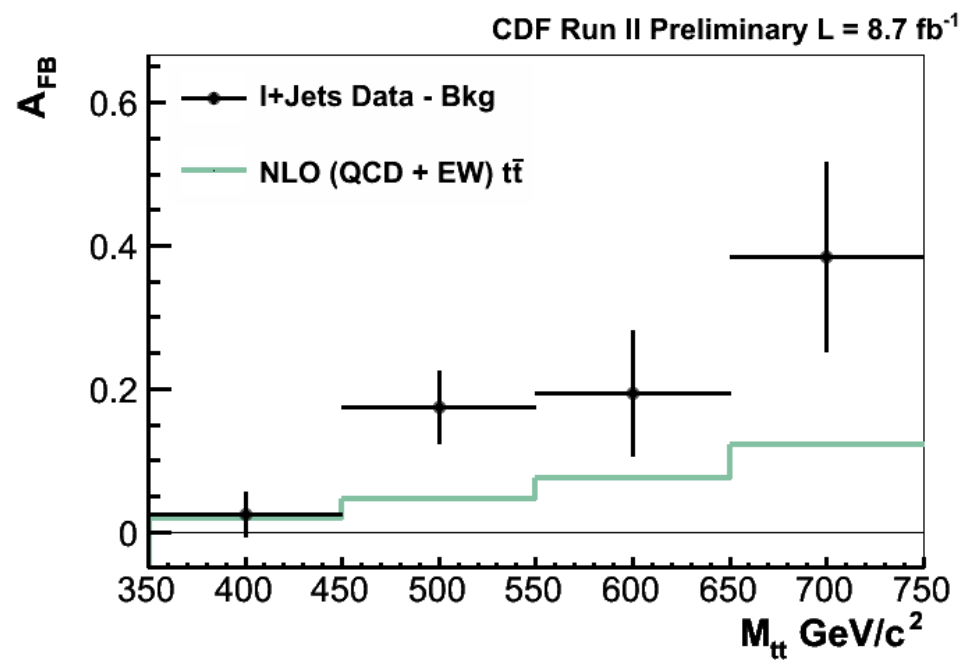

Figure 11: Dependence of $A_{F B}$ on $M_{t t}$ for the data from CDF after the background subtraction. Beside the lowest mass point, which is consistent with SM prediction, the other three data points show discrepancy with SM.

After corrections for the bias in the trigger and in event reconstruction, the distribution of the $\boldsymbol{t} \overline{\boldsymbol{t}}$ differential cross section as a function of $\Delta y$ is shown in Figure 12. The forwardbackward asymmetry of the $t \overline{\boldsymbol{t}}$ system is measured to be $0.162 \pm 0.047$ from the full data of CDF. For D0, the asymmetry is measured to be $0.196 \pm 0.065$ using data of $5.4 \mathrm{fb}^{-1}$ luminosity. 


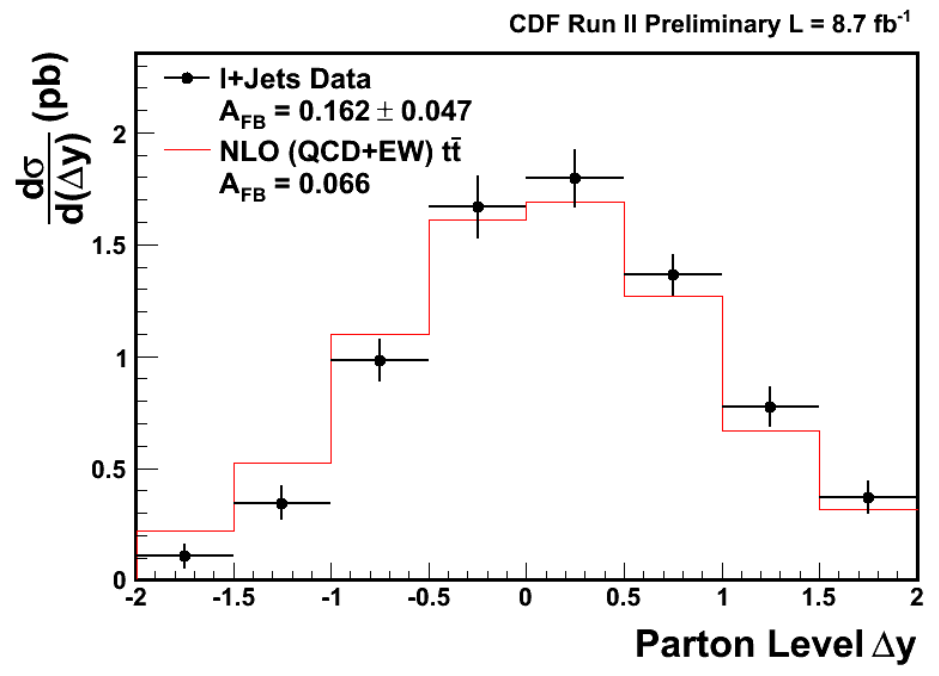

Figure 12: The differential cross section of the $t \bar{t}$ quark production as a function of $\Delta y$.

\section{Conclusion and comment}

Both the CDF and D0 observe an asymmetry in the angular distribution of $t \bar{t}$ quarks. The dependence on $\Delta y$ and on the mass of the $t \bar{t}$ system is observed by CDF, but with somewhat less significance by D0. More studies from both experiments are anticipated to include the differential cross section as a function of $\cos \theta^{*}$, and additional result from the DIL channel.

The results on $A_{F B}$ from ATLAS and CMS show consistency with the SM prediction [10]. However there is fundamental difference between the LHC and Tevatron. The $t \bar{t}$ pairs produced at the LHC proceed mostly through gluon-gluon fusion, while at the Tevatron, the production are mainly from quark antiquark annihilation. Because of $p p$ symmetry, no angular asymmetry is possible for either top or antitop quark in the $t \bar{t}$ rest frame. The LHC experiments must study the top and antitop quarks in the high rapidity region where there is good fraction of top quark pairs from quark anti-quark annihilation. This would be a fair comparison with $A_{F B}$ from CDF and D0. Should there be significant deviation from SM prediction that would either increase our understanding of QCD or there might be chance for new physics.

\section{References}

[1] The CDF collaboration, Observation of Top Quark Production in $\mathrm{p}$ anti-p Collisions with the Collider Detector at Fermilab, Phys. Rev. Lett. 74, 2626 (1995).

[2] The D0 collaboration, Observation of the Top Quark, Phys. Rev. Lett. 74, 2632 (1995).

[3] The CDF collaboration, Measurement of the top pair production cross section in the dilepton decay channel in $p \bar{p}$ collisions at $\sqrt{\mathrm{s}}=1.96$ TeV, Phys. Rev. D 82, 052002 (2010).

[4] The D0 collaboration, Measurement of the t站Production Cross Section using Dilepton Events in pp_Collisions, Phys. Lett. B 704, 403 (2011)

[5] M. Beneke, P. Falgari, S. Klein, J. Piclum, C. Schwinn, NNLL threshold resummation for the total top-pair production cross section, arXiv: 1205.0988 [hep-ph]. 
[6] The D0 collaboration, Measurement of the Top Quark Pair Production Cross Section in the Lepton + Jets Channel in Proton-Antiproton Collisions at $\sqrt{s}_{s}=1.96 \mathrm{TeV}$, Phys. Rev. D 84, 012008 (2011).

[7] The CDF collaboration, Evidence for a mass dependent forward-backward asymmetry in top quark pair production, Phys. Rev. D 83, 112003 (2011).

[8] The D0 collaboration, Forward-Backward Asymmetry in Top Quark-Antiquark Production, Phys. Rev. D 84, 112005 (2011).

[9] J. A. Aguilar-Saavedra, M. Perez-Victoria, Asymmetries in tt production: LHC versus Tevatron, Phys. Rev. D 84, 115013 (2011).

[10] Shown at this conference. 